\title{
Pensieri all'aperitivo. \\ Imparare a padroneggiare il sistema di numerazione decimale posizionale in situazioni divertenti
}

\author{
Thinking during the aperitif. \\ Learning to master the decimal place value system in entertaining \\ situations
}

\author{
Stefan Meyer \\ Interkantonale Hochschule für Heilpädagogik - Zurigo, Svizzera \\ 凶stefan.meyer@hfh.ch
}

QUESTO ARTICOLO Ė DISPONIBILE ANCHE IN LINGUA ORIGINALE

Sunto / L'articolo descrive come è possibile sviluppare il pensiero logico-matematico e le competenze operative concernenti il sistema di numerazione decimale posizionale in modo creativo, operativo ed esemplificativo. Teoricamente l'articolo è vicino al costrutto di «accelerazione cognitiva» (Adey, 2008) che collega in una "scuola del pensiero" la teoria genetica dello sviluppo cognitivo (Piaget, 1977a, 1977b; Piaget \& Voelin, 1980), il Piano di studio in vigore (Lehrplan21), la zona di sviluppo prossimale (Vygotskij, 1986) e la metacognizione. Inoltre, l'articolo integra il gioco di ruolo nel senso di esperimento sociometrico (Moreno, 1996; 2007), il metodo dell'esplorazione critica (Piaget, in Inhelder, Sinclair \& Bovet, 1974) e l'abaco scolastico come mezzo operativo di rappresentazione del sistema di numerazione decimale posizionale (Johann, 2002; Johann \& Matros, 2003). Questi metodi possono orientare il processo di comprensione durante l'apprendimento della matematica.

Parole chiave: aritmetica; sistema di numerazione decimale posizionale; abaco; mezzo di rappresentazione; gioco di ruolo.

\begin{abstract}
The paper describes how it is possible to develop logical-mathematical thinking and operational skills regarding the positional decimal numeral system in a creative, operational and illustrative way. Theoretically the paper is close to the construct of «cognitive acceleration» (Adey, 2008) that connects in a "school of thought" the genetic theory of cognitive development (Piaget, 1977a, 1977b; Piaget \& Voelin, 1980), the current curriculum (Lehrplan21), the proximal development zone (Vygotskij, 1986) and metacognition. Moreover, the article integrates the role play in the sense of sociometric experiment (Moreno, 1996; 2007), the method of critical exploration (Piaget, in Inhelder, Sinclair \& Bovet, 1974) and the abacus as an operational tool of representation of the positional decimal numeral system (Johann, 2002; Johann \& Matros, 2003). These methods can guide the understanding process during the learning of mathematics.
\end{abstract}

Keywords: arithmetic; positional decimal numeral system; abacus; tool of representation; role play. 


\section{Il problema}

Il sistema di numerazione decimale posizionale è una rappresentazione concettuale e simbolica dei numeri naturali $(\mathbb{N})$ fino ai reali $(\mathbb{R})$. Secondo Reiss e Schmieder (2005) è un sistema semplice, ma allo stesso tempo anche impegnativo; in effetti giungere alla comprensione del nostro sistema di numerazione è un compito complesso che richiede molto tempo. I risultati della ricerca degli ultimi decenni confermano che la concezione di numerosità, normalmente acquisita da alunni e alunne tra il secondo e il quinto anno della scuola elementare, in alcuni casi si protrae fino alla secondaria e oltre (Ross, 1986; Schuler, 2004; Brugger, Sidler \& Meyer, 2007; Moser Opitz, 2007; Ruflin, 2008; Herzog, Fritz \& Ehlert, 2017; Herzog, Ehlert \& Fritz, 2019; vedi Tabella 1).

Dai dati delle ricerche cliniche relative allo sviluppo psicologico del pensiero è difficile dedurre informazioni sullo sviluppo dei livelli cognitivi raggiunti dagli allievi, perché di solito le ricerche sono effettuate in ambiti creati ad hoc. Anche nel caso di dati provenienti da ricerche in classe, occorre chiarire a quale modello pedagogico appartengono: sono compiti artificiali ed esercizi ripetitivi, o sono problemi reali che suscitano interesse? Dati sull'apprendimento dipendono dunque dalla qualità dei processi formativi realizzati dai docenti in ambito matematico e da altri fattori correlati (Dewey, 2008; Weltgesundheitsorganisation, ${ }^{1}$ 2011). Secondo Dewey (2008), è possibile valutare un costrutto matematico come il sistema di numerazione decimale posizionale, oggetto dell'articolo, sviluppato con o senza riferimento a situazioni; questo perché «[...] non consideriamo mai le esperienze di oggetti o eventi per sé stessi, nemmeno formuliamo valutazioni su giudizi formulati unicamente su di essi, ma solo in un contesto nel suo insieme» (Dewey, 2008, p. 87, traduzione dell'autore). Analogamente Kamii $(1985,1994,2004,2005)$ sottolinea in modo rigoroso che il pensiero logico-matematico non può essere insegnato, ma conquistato in prima persona e co-costruito. Inoltre, Wittmann (2002) ha sviluppato il concetto dell'insegnamento e dell'apprendimento attivo ed esplorativo, ma si è reso conto che tutti i nobili approcci didattici vengono vanificati dalla pressione dei tempi e dall'uso dei materiali didattici considerati. Così la formazione è in contrasto non solo con i problemi che scaturiscono dal sistema di numerazione stesso, ma anche con i limiti di insegnamento-apprendimento interni alle situazioni assunte.

Alla luce della pedagogia critica (Gur-Ze'ev, 2005; Freire, 2011; Wink, 2011), i limiti del lavoro dell'insegnante hanno a che fare con la pedagogia della trasmissione, che è il credo che in parte continua ancora a esistere nella pratica di tanti insegnanti. Questa pedagogia si attua attraverso una "didattica dei compiti", ossia una didattica basata sull'esclusiva consegna di esercizi staccati da un contesto reale, che è stata analizzata da Lenné (1969, "Aufgabendidaktik"). Secondo Freudenthal (1977), i presupposti della pedagogia della trasmissione si manifestano nei compiti focalizzati esclusivamente su riferimenti intra-matematici, senza un aggancio con la realtà circostante degli allievi, ossia con riferimenti extra-matematici. La conseguenza è, secondo Freudenthal, che la didattica diventi un sistema illusorio (intra-matematico). La distinzione proposta da Freudenthal serve per capire a fondo i concetti e i design moderni. Secondo Freudenthal, le relazioni extra-matematiche sono la linfa vitale dell'educazione, comprese le situazioni didattiche. Freudenthal (1991) creò il ben noto concetto didattico "Realistic Mathematics Education" (RME) con il quale vengono superate le tentazioni del sistema illusorio. L'aggettivo "realistic" indica l'esperienza dell'allievo con la matematica scolastica agganciata con la matematica vissuta nella propria vita quotidiana. Allo stesso tempo il significato di "realistic" tende ad esplorare e integrare la matematica nella mente degli allievi attraverso dei vivi processi educativi e di reinvenzioni guidate. Per definizione la didattica dei compiti non integra la sintesi realistica e mentale degli allievi, rimanendo un "ping-pong behavioristico" fra stimolo e reazione (Abrahamson, Zolkower \& Stone, 2020).

1. Organizzazione per la salute mondiale. 
Il sistema illusorio può essere esaminato e superato grazie a metodi sociometrici come quello proposto da Moreno (1996) e che vengono presentati in questo articolo. II credo nella pedagogia della trasmissione, la concentrazione sulle relazioni esclusivamente interne alla matematica, come anche la didattica dei compiti, sono indicatori dell'illusione e della mancanza di relazioni esterne al mondo intrinseco della matematica. Secondo Moreno (1996) esiste una tendenza paradossale fra innovazione, produzione e consumo, secondo la quale prodotti innovativi, creati con processi creativi, vengono dati in pasto a usi routinari e consumistici; nel caso specifico della matematica, materiali didattici innovativi vengono "imprigionati" nell'attuale didattica dei compiti. Moreno chiama questi prodotti "conserve culturali» (Storch, 1996; Moreno, 2007, in inglese: "cultural conserves»). Storch (1996, p. 1, traduzione dell'autore) riprende il concetto e precisa: «Le conserve rappresentano il tentativo di congelare la spontaneità e la creatività di un momento passato in un prodotto concreto».

Se descrizioni e processi come la semplice trasmissione di contenuti scolastici diventano dominanti nel processo di insegnamento-apprendimento della matematica, indipendentemente dalla comprensione della materia, è evidente che la spontaneità come requisito della creatività viene del tutto persa (Storch, 1996).

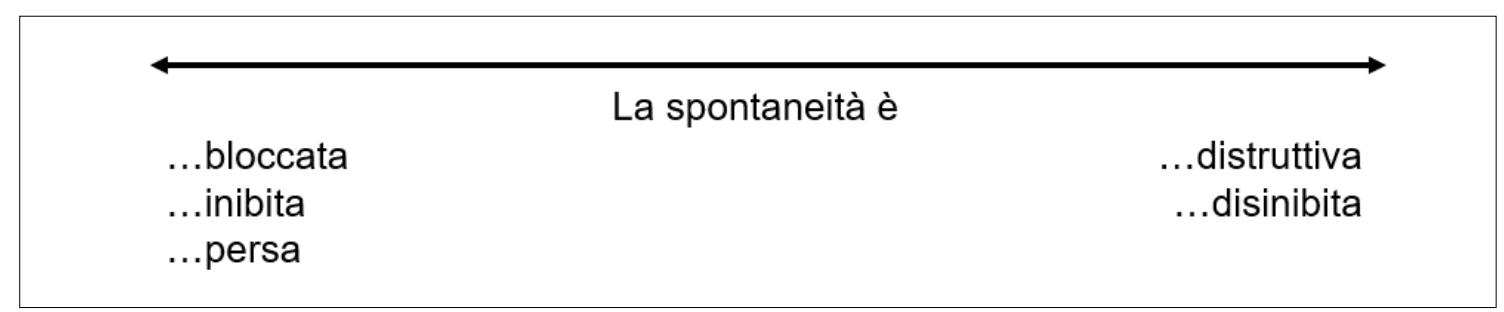

Figura 1. Poli della nevrosi della creatività.

La Figura 1 mostra schematicamente, secondo ciò che sostengono Wieser e Ottomeyer (2000, p. 662), come le capacità di agire e socializzare vengono bloccate o disinibite da un approccio nevrotico alla creatività che conduce a polarizzare anche la spontaneità, che può così emergere in modo alternato e passare dall'essere inibita o, all'opposto, completamente disinibita. Tradotto in ambito formativo, ciò significa che pratiche didattiche che sacrificano la comprensione dei contenuti in gioco mettono in pericolo anche la spontaneità di chi le vive. Coloro che si aggrappano ad una didattica immediata, scontata e "dozzinale" (Freire, 2011) considerano la spontaneità come un disturbo all'apprendimento della matematica e seguono quello che Watzlawick (2017) chiama "gioco senza fine». All'interno di questa dinamica esistono attività sociali nelle quali i partecipanti non capiscono le regole di ciò che si sta facendo come avviene, per esempio, per la pedagogia della trasmissione. Per superare tali dinamiche, Watzlawick sostiene la necessità di un salto nell'astrazione degli apprendimenti e un approfondimento dell'errore. Con ciò la spontaneità e la creatività potrebbero fare da censura della pedagogia della trasmissione aiutando a superare la paura irrazionale nei confronti della matematica e favorendo la sua comprensione (Devereux, 1998).

Non basta quindi considerare la didattica della matematica come «design science» (Wittmann, 2020, p. 66), ossia come modernità fine a sé stessa: occorre invece che la «design science» conosca e padroneggi il paradosso delle "conserve culturali" e dei "giochi fini a sé stessi".

Alcuni metodi non conformisti possono permettere questo salto di prospettiva. Secondo Freudenthal $(1983,1991)$ tali metodi potrebbero essere ancorati all'analisi didattica (Klafki, 1996; S. Meyer, 2020a) che è un processo multidimensionale pensato per guidare la preparazione delle lezioni. In questo approccio, gli insegnanti organizzano e perfezionano l'insieme di queste dimensioni: le risorse 
e le condizioni, i contenuti significativi (che sono gli elementi realistici secondo Freudenthal, 1991), gli scopi, i metodi, la comunicazione, la cooperazione e l'interazione, e l'organizzazione delle sequenze. Più vivi e sistemici risultano gli scambi tra le persone e i diversi elementi in gioco, più significativo risulta il processo educativo. In un design di questo tipo, emerge la spontaneità come adeguata reazione di fronte a una nuova situazione o come nuova reazione davanti a vecchie situazioni (Wieser \& Ottomeyer, 2000).

Per favorire la spontaneità risulta interessante anche la metodologia dei giochi di ruolo.

In un progetto relativo allo sviluppo dell'alfabetizzazione tramite il racconto di storie e i giochi di ruolo, Gupta (2009) riscontrò i seguenti risultati:

1. I bambini svilupparono un ricco vocabolario e strutture di frasi complesse.

2. I bambini mostrarono profonde attitudini emozionali nel gioco di ruolo. Si confrontavano con i propri conflitti sociali, portandoli a un maggiore apprezzamento di sé stessi e a un innalzamento della fiducia nelle proprie capacità.

3. Le questioni sociali relative a giochi di genere, impegno, problemi di etica e altri aspetti relativi all'esperienza vissuta furono gestite con grande consapevolezza e interesse.

4. I giochi di ruolo consentirono di risolvere problemi in comune, di negoziare, organizzare e decidere.

5. Nei colloqui peer to peer i bambini si sostenevano a vicenda nel pensiero fattuale e sociale.

6. Aumento della capacità di riconoscere diversi punti di vista e possibilità. L'io egocentrico diventò così decentrato. Si sono pure sviluppati il senso di comunità e il fatto che il sapere del gruppo si pone al di sopra di quello dei singoli.

L'idea centrale del gioco di ruolo fu elaborata successivamente da LeMa-Methode (Leggere e matematizzare) nel MKT-Testsystem (S. Meyer \& Wyder, 2017), che ha rilevato l'efficacia di tale metodo. I risultati hanno messo in evidenza come sia particolarmente utile che gli insegnanti, di fronte a problemi di calcolo concernenti l'insegnamento dell'aritmetica, si calassero nel ruolo di una segretaria spiritosa e lasciassero al bambino il "ruolo di protagonista" nella risoluzione di problemi. In particolare, nel caso specifico di una bambina di seconda elementare, convinta che non sarebbe mai riuscita ad effettuare calcoli, sono stati osservati effetti molto positivi. La docente di sostegno giocò in questo caso il ruolo di calcolatrice tascabile e tramite questo gioco di ruolo la bambina intavolò subito con lei un rapporto costruttivo e competente (S. Meyer, 2019).

La spontaneità, nel senso della sociometria, è requisito per nuove e creative forme di insegnamentoapprendimento, che saranno delineate in seguito relativamente al sistema di numerazione decimale posizionale.

\section{Pensieri pedagogico-didattici}

Supponiamo di dover organizzare un aperitivo verso le 11 del mattino. Nel caso di attività concordate con un significato sociale (Klafki, 1996; S. Meyer, 2020a), la risoluzione di problemi matematici, il pensiero operativo e socialmente condiviso e l'astrazione riflessiva (Piaget, 1977a, 1977b) sono promossi con creatività. Problemi attinenti al sistema di numerazione decimale posizionale, e concernenti idee errate, conflitti cognitivi o nuovi interessi potrebbero essere trattati con l'abaco scolastico o con, per esempio, bastoncini salati nel ruolo di mezzi di rappresentazione. Alla fine del rituale i bastoncini vengono mangiati e la classe si concede un bicchiere d'acqua o di sciroppo. 
L'esplorazione del fenomeno matematico si svolge in un processo co-costruttivo del gruppo. I risultati vengono messi in comune nel plenum, ossia nell'informazione e nell'argomentazione di tutti (sharing) e anche discussi in senso metacognitivo. Alla fine di questa prima fase chiamata "bridging", si pone l'attenzione sull'insegnamento che avviene nel tempo libero e nel nuovo rituale di aperitivo. "l "bridging" crea dei ponti verso i rituali futuri.

Questi elementi metodici appartengono al fondamento del supporto all'apprendimento nel senso di Vygotskij (1986) e all' «accelerazione cognitiva» (Adey, 2008). Le possibilità di rappresentazione (dal concreto al sistema di scrittura numerico-algebrico), come anche le operazioni fatte con l'ausilio di mezzi di rappresentazione, danno informazioni sul livello attuale del pensiero astratto e anche sui livelli del leggere e capire relativi al sistema di scrittura matematico.

La tecnica sviluppata dalla scuola ginevrina della «vérification sur le vif» e l' «astrazione riflessiva» (Inhelder, Sinclair \& Bovet, 1974)² sono molto vicine alla zona di sviluppo prossimale di Vygotskij (1986). L'ambito è quello della verità logico-matematica di modelli e modi di pensiero e di azione. Vengono esaminati ipotesi di contenuti e connettivi e in seguito tematizzati processi di un'intervista flessibile o di un episodio nell' "accelerazione cognitiva», oppure testati operativamente e socialmente. Ciò stimola l'astrazione riflessiva, come hanno dimostrato le ricerche sull'accelerazione cognitiva: gli alunni diventano intellettualmente più pronti, dimostrando l'efficacia dell' «accelerazione cognitiva», come fattore ambientale (Inquiry-Situation; vedi Adey, 2008).

\subsection{La progettazione della conversazione nell' "aritmetica ludica"}

In questo articolo viene anche collegato il metodo di «esplorazione critica» (Piaget in Inhelder, Sinclair \& Bovet, 1974), che è una parafrasi dell'intervista flessibile, con la co-costruzione e la zona di sviluppo prossimale di Vygotskij (Gupta, 2009). In tale prospettiva sono integrati anche la metacognizione (Piaget, 1977a, 1977b; Adey, 2008) e i metodi di azione di Moreno (1996, vedi Figura 2; H. Meyer, 2000).

\section{Metodi sociometrici \\ Metodi di gruppo \\ Test sociometrico \\ Test di conoscenza \\ Questionario sociometrico \\ Metodi di azione \\ Test di spontaneità \\ Test del gioco di ruolo \\ Test della situazione \\ Psicodramma \\ Sociodramma \\ Cambio di ruolo \\ Metodi di auto-conversazione \\ (doppi, a specchio, simulati)}

Figura 2. Dimensioni del metodo sociometrico secondo Moreno (1996).

2. La «vérification sur le vif», che è una delle caratteristiche fondamentali dei nostri metodi, procede a passi dall'esplorazione critica e colloquiale del bambino all'analisi e all'interpretazione dei modi di comportamento (Inhelder, Sinclair \& Bovet, 1974, p. 40; traduzione dell'autore). 
I metodi sociometrici (Figura 2) indicano varie possibilità di azione che qui saranno discusse parzialmente. La spontaneità bloccata e la creatività ristretta nelle «conserve culturali» (Moreno, 2007) vengono liberate dai giochi e dall'esplorazione critica. In tal senso, I'approccio al sistema di numerazione decimale posizionale è introdotto e inscenato mediante il teatro improvvisato, il cambio di ruoli, i soliloqui, i giochi di pensiero, "I'artista aritmetico", espressione che deriva dal greco "arithmós" (numero) e dall'aggettivo arithmētikós (appartenente al conteggio o al calcolo) e da téchnē (arte), cioè l'arte del conteggio o del calcolo, e anche da colloqui di gruppo. Di qui si arriva direttamente al «calcolo ragionato» come rappresentazione ragionata di pensieri e fatti. I metodi di azione sono sempre correlati con domande o richieste.

Arrigo (2014) riferisce ciò che intende con «calcolo ragionato» in relazione alla teoria delle trasformazioni semiotiche di Duval (1993; S. Meyer, 2017; vedi par. 4). II processo è fondamentalmente mentale: il calcolo pensato e argomentato, con più gradi di libertà, si conforma in calcolo mentale con supporto scritto. Questo metodo ingloba la concettualizzazione (intuizione del concetto), I'apprendimento strategico, la comunicazione e l'approccio alla rappresentazione. II calcolo ragionato o arte riflessiva dei numeri si trasforma in pratica in un'introduzione ben fondata nelle operazioni di base e nelle leggi commutative, associative e distributive del calcolo (Arrigo, 2014).

La dinamica di questi metodi contribuisce al superamento della pedagogia della trasmissione e dell'istruzione inefficace. I metodi portano motivazione e promuovono la comprensione dei concetti matematici e relative connessioni, anche quando sorgono problemi di comprensione, conflitti sociali o cognitivi. Le soluzioni non sono ricercate razionalmente, ma attraverso esplorazioni ludiche e rassicuranti. II dilemma relazioni intra-matematiche/extra-matematiche (Freudenthal, 1977) diventa elemento dinamico dell' "aritmetica ludica", del gioco di ruolo matematico.

\subsection{Matematica mentale e comunicazione liberatoria}

I metodi e i principi descritti sopra sono certamente complessi. Quali atteggiamenti e quali tecniche del colloquio sono necessari, per far sì che docenti professionisti diventino liberi comunicatori e moderatori di tali pratiche? I seguenti suggerimenti facilitano l'acquisizione di pratiche efficaci della comunicazione (Cuomo, 2007; Gupta, 2009):

- Proporre a bambini o adulti uno stimolo che può essere pensato liberamente o integrare un problema mediante un colloquio con alunne e alunni.

- Proporre uno stimolo invitante e che porti gioia.

- Lo stimolo può rappresentare parte di un gioco di ruolo. Tutti si comportano in modo conveniente, come a un aperitivo nel quale si discute su una questione interessante.

- Gli stimoli funzionano attraverso modelli, giochi di ruolo e teatro improvvisato. Persone o figure (peluches) sono gli attori. Potrebbero esordire con affermazioni come: "L'orso (o Elsa) ha un po' di fame, al ristorante (della famiglia o della scuola) è servito un aperitivo. Vengono offerti sciroppo e bastoncini salati, ma di questi ce ne sono solo tre». (In particolare, un bastoncino su una carta che rappresenta le decine e due bastoncini sulla carta delle unità). Può anche essere organizzato un vero rituale di aperitivo.

- Non affidare agli allievi troppi compiti alla volta. Si potrebbe fare come nel calendario dell'avvento. Invece del Natale si aspetta l'entrata nel sistema di numerazione decimale posizionale.

- Trattenersi dal dare istruzioni e assumere un atteggiamento socratico.

- Assumere con delicato humor il ruolo di una persona smemorata e irragionevole e stimolare gli alunni alla riflessione e alla comunicazione.

- Non spiegare mai passo per passo e linearmente. L'intuizione non sorge in modo lineare, ma attraverso un pensiero cosciente e anche incosciente (sentire) attraverso canali nervosi collegati esponenzialmente. 
- Proporre molte figure e lasciare scegliere ai bambini quelle che preferiscono. Ci sono molti modelli di ruolo interessanti come per esempio «La Linea - Series» (Osvaldo Cavandoli) oppure «ll rosso e il blu» (Francesco Misseri: https://www.youtube.com/watch?v=koZeA5zDbF0) oppure Mio Mao (Misseri Studio: https://www.youtube.com/user/MISSERISTUDIO/videos).

- Durante questi giochi occorre essere vivaci e proporre una dopo l'altra domande e interventi matematici. Il compagno di gioco o i bambini vengono incoraggiati a comunicare. Le domande e i problemi circolano. Non deve nascere alcun effetto "ping-pong" tra alunno e insegnante.

- Le cosiddette "lacune dell'apprendimento» vengono integrate e collegate dall'intuizione. Occorre avere fiducia e confidare apertamente nella crescita delle risorse del gruppo classe.

- Osservare liberamente come farebbe un barista discreto.

- Porre spontaneamente domande aperte e pertinenti, che invitano gli altri a pensare, parlare e agire.

- Invitare i gruppi a descrivere con segni e pensieri diversi (Duval, 1993; Presmeg, Radford, Roth \& Kadunz, 2016). I segni e i pensieri possono essere espressi oralmente, per iscritto, con disegni oppure in modo enattivo, ossia attivo-motorio (Bruner, 1971). Fare circolare le domande sul significato dei segni.

- Evitare giudizi del tipo errato-corretto. Chiedere invece sempre a ciascuno: «È vero?» (nel senso della verità logico-matematica). "Da cosa deduci che le tue conclusioni sono vere? Su cosa ti basi quando giudichi se quello che ha detto un tuo compagno o una compagna è vero?»

- Esigere sempre una giustificazione, come per esempio potrebbe sostenere l'orso: "Ma questo non può essere vero! Trovate dimostrazioni che mi possano convincere». Come detto sopra, è I'orso (o Elsa) che dice queste cose. Se ci si tuffa nel gioco di ruolo, questo stile comunicativo allegro e dinamico riesce meglio (Kamii, 2000; Bodrova, 2007; Cuomo, 2007; Wittgenstein, 2013; Resnick, Asterhan \& Clarke, 2018; Schenker, 2018; Piaget in Inhelder, Sinclair \& Bovet, 1974; Piaget, Henriques \& Ascher, 1990).

- Gestite questi conflitti cognitivi, per esempio dicendo: «Su questo possiamo rifletterci domani. Se ci troviamo per l'aperitivo possiamo proporre la stessa questione ancora una volta? Lo volete? L'orso ne ha abbastanza!» Con ciò si crea una tensione mentale motivante nei confronti del problema matematico. Come già anticipato, occorre non distruggere la tensione cognitiva dando la soluzione!

I suggerimenti chiariscono il fatto che il gioco di ruolo matematico è una metodologia divertente e intelligente. Secondo Freire (2011) ci si muove nel campo dell'empatia e non nel campo della comunicazione subita. Il gioco di ruolo crea così uno stile di educazione vissuto e positivo (Cuomo, 2007), nel quale è normale trovarsi in situazioni senza capire e in cui l'apprendimento diventa empatico e bello. L'aritmetica vissuta proposta dall'insegnante rappresenta così un modello che si colloca nella zona dello sviluppo prossimale nel senso di Vygotskij (1986).

\section{L’aperitivo come esperimento pedagogico, la PASS-Teoria}

La cura dell'intuizione nel sistema di numerazione decimale posizionale e nelle relative rappresentazioni richiede uno sforzo di più anni con problemi e riflessioni astratte sul compito stesso (Inhelder, Sinclair \& Bovet, 1974; Piaget, 1977a, 1977b; Adey, 2008). Sulla linea di Dewey (2008) occorre scoprire gli oggetti del sistema di numerazione all'interno di una situazione. II rituale dell'aperitivo, inteso come situazione, discende dalla neuropsicologica PASS-Teoria sviluppata da Lurija. Si tratta di una serie di compiti e criteri, che sono stati inclusi nei test proposti da Goldstein, Princiotta e 
Naglieri (2015) e specialmente da Otero (2015). Lurija e Judowitsch (1982), e Cuomo (2007) hanno trasformato la logica dei test nella logica dei processi della formazione scolastica integrativa. L'analisi didattica e sistemica descritta nel primo paragrafo serve come avvocato-mediatore (Klafki, 1996; S. Meyer, 2020a).

PASS è l'acronimo di Piani dell'Áttenzione, della Simultaneità e della Successione, che in relazione al rituale dell'aperitivo significano:

- Piani. Sono processi cognitivi, operativi e sociali, nei quali persone in situazioni si sfidano a determinare, scegliere, applicare strategie e tattiche nella risoluzione di problemi. Pianificare è perciò compito dei professionisti e dei bambini, che, al servizio dell'intuizione, sono messi di fronte al sistema di numerazione decimale posizionale. Questi processi sono annoverati fra le funzioni esecutive.

- Attenzione. È un processo cognitivo e sociale che è richiesto a persone che trattano selettivamente un certo contenuto o una determinata domanda. Questo processo impedisce che la persona venga ostacolata da contenuti concorrenti.

- Simultaneità. È un processo cognitivo, operativo e sociale, nel quale stimoli separati o contenuti vengono integrati in un unico insieme o gruppo. Questa è una caratteristica fondamentale del rituale aperitivo nel quale il sistema di numerazione viene discusso simultaneamente con tutti i mezzi e le conoscenze.

- Successione. È un processo cognitivo, operativo e sociale, che ordina serialmente cose/fatti: ad esempio, mettere acqua nella Moka significa qualcosa d'altro che mettere la Moka nell'acqua, così come scrivere 12 significa qualcosa di diverso che scrivere 21; "Prima la moltiplicazione" è un motto che regola la successione (la precedenza) nelle operazioni aritmetiche. Nell'aperitivo troviamo successioni della simbolizzazione, della descrizione, delle parentesi, della quantificazione come anche il rituale del «prima il dovere e poi il piacere!».

Fondamenti neuropsicologici e pedagogici dei rituali nella PASS-Teoria sono la stimolazione ottimale, le emozioni e l'attenzione (Lurija \& Judowitsch, 1982; Cuomo, 2007; Goldstein, Princiotta \& Naglieri, 2015; Otero, 2015). La PASS-Teoria permette una relazione dinamica e olistica tra insegnamento e apprendimento del sistema di numerazione nei rituali dell'aperitivo o in altri colloqui matematici. L'attenzione condivisa, I'organizzazione e i piani comuni delle attività in riferimento ai problemi cognitivi e ai compiti garantiscono il successo nell'apprendimento (Dewey, 2008; McCabe \& Farrell, 2020).

\section{Riflessioni sulla teoria delle rappresentazioni (Duval, 1993)}

Secondo Duval (1993), quando si ha a che fare con un oggetto matematico si dovrebbero mobilitare più registri semiotici e diverse forme di rappresentazione (citato anche in Presmeg et al., 2016) (vedi Figura 3). 

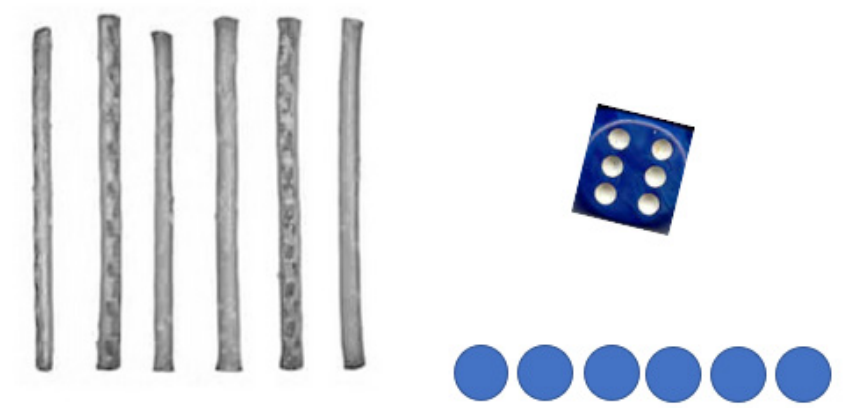

\section{$\begin{array}{lllllllllllll}1 & 1 & 1 & 1 & 1 & 1 & 1 & 1 & 1 & 1 & 1 & 1\end{array}$ 6 bastoncini salati 6 piattini}

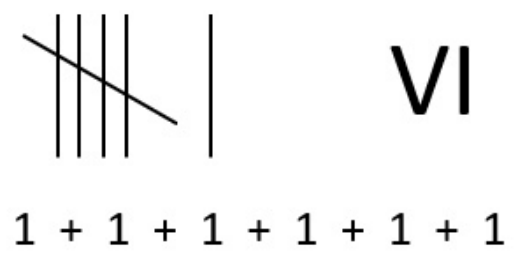

$$
3+3
$$$$
12: 2=6
$$

Figura 3. Esempi di rappresentazioni del numero 6.

La diversità dei registri semiotici (linguaggi) in classe è sempre presente, quando i bambini possono mostrare liberamente le loro rappresentazioni e i linguaggi scelti, se non sono obbligati a seguire sistemi di segni (linguaggi) convenzionali, con i quali il pensiero viene presentato come fatto [figure, grafici, scritture simboliche, l'insieme delle cifre da 0 a 9, il linguaggio comune ecc. (vedi Duval, 1993, p. 40)]. Per contro, i diversi registri semiotici contribuiscono a far capire che gli oggetti matematici non possono essere scambiati con la loro rappresentazione, ma è proprio l'uso di tutte queste rappresentazioni che contribuisce alla costruzione dell'oggetto matematico.

Secondo Duval (2006, p. 64) la moltitudine delle possibili rappresentazioni genera un problema cognitivo necessario per I'apprendimento. II problema cognitivo emerge per esempio quando si mostrano le diverse rappresentazioni (espressioni simboliche) di 100: 90+10; 5.20; 1000:10; 1: $\frac{1}{100} \ldots$, in quanto occorre trovare delle proprietà invarianti nelle molteplici forme di rappresentazione di un oggetto. "La matematica è ricerca di invarianti» (comunicazione orale di Hans Walser, 2020-01-17 dedicata al suo professore di matematica Heinz Hopf): apprendere significa avanzare in mezzo a problemi cognitivi verso la conoscenza delle cose comuni, cioè sapere cosa è comune e perché è comune (vedi anche Piaget \& Szeminska, 1975). Di conseguenza, se si fornisce agli allievi solo un'univoca rappresentazione dell'oggetto in gioco e si prescinde da buone intenzioni didattiche, tale rappresentazione uniformata genera sistematicamente disturbi e un effetto paradossale, precludendo così la possibilità agli insegnanti di integrare la matematica vissuta dagli allievi (vedi la nozione "realistic" di Freudenthal nei par. 1 e 2.1).

\subsection{L'abaco scolastico come sistema di rappresentazione}

Johann (2002) e Johann e Matros (2003) hanno presentato l'abaco scolastico come mezzo di rappresentazione. Essi mostrarono con esempi concreti che questo strumento risulta molto utile anche per alunni con difficoltà cognitive nell'ambito del sistema di numerazione decimale posizionale. Vantaggi 
di questo mezzo sono l'adattabilità e le infinite possibilità d'uso. È un mezzo didattico e nello stesso tempo una metodologia qualitativa di ricerca, nel senso che gli allievi operano con le carte posizionali e con i simboli per le cifre, spiegando ed esplorando la logica delle strutture aritmetiche, mentre l'educatore-ricercatore provoca la conversazione sul significato del fare e del dire. Questo metodo si chiama "Strukturlegetechnik" (The structure laying technique; Flick, 2006) e si situa molto vicino al metodo dell'esplorazione critica di Piaget. In questa attività gli alunni usano fogli sciolti e barrette (di legno, o anche bastoncini salati) per fissare quantità nel sistema posizionale con la base 10 (Barrow, 1999). Queste quantità possono essere scritte in vari modi e convertite tra rappresentazione iconica e simbolica. II materiale è variabile, i simboli possono essere spostati, rielaborati, scambiati e combinati. Gli alunni discutono e verificano le rappresentazioni ottenute. Eventualmente le rappresentazioni possono essere anche fotografate, se devono essere ulteriormente elaborate. La struttura del materiale è funzionale a poter rappresentare e chiarire meglio il sistema posizionale: gli alunni scoprono la logica della scrittura dei numeri nel nostro sistema (Barrow, 1999, p. 162), ciò che con Freudenthal (1991) può essere indicata come "guided-reinvention».

È possibile iniziare l'esperienza prendendo 2-3 fogli A4 e scrivendo ciò che viene proposto nella Figura 4. È possibile proporre ai bambini un gioco di ruolo, nel quale il docente scrive dei numeri e loro devono individuare il criterio adottato.

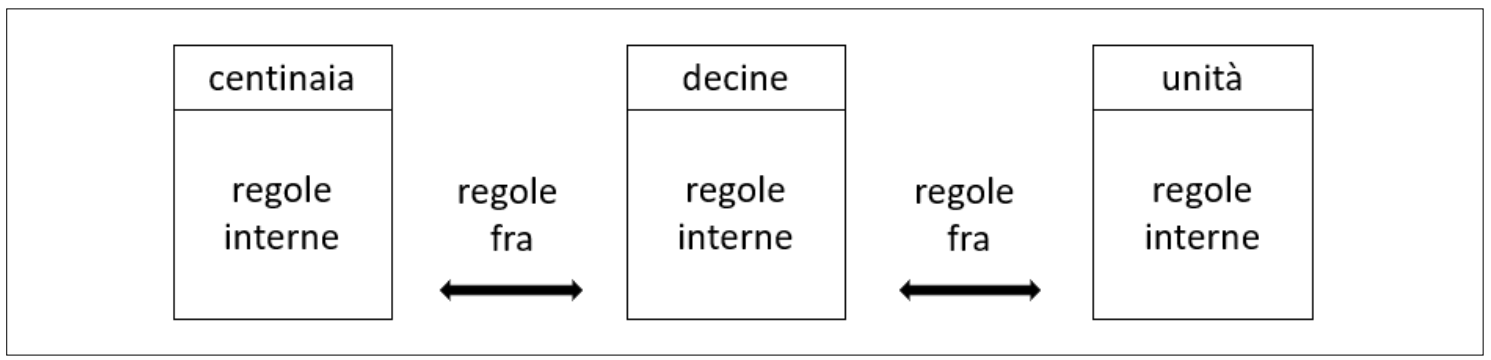

Figura 4. Rappresentazione schematica del sistema di numerazione posizionale con base 10.

L'approccio alla logica del sistema di numerazione decimale posizionale proposto nella Figura 4 diventa difficile se gli alunni devono solamente applicare regole date (Barrow, 1999; Reiss \& Schmieder, 2005). La base 10, la successione delle potenze di 10, il significato del numero 0 , le operazioni aritmetiche, le regole del calcolo (soprattutto le leggi associativa e distributiva) e le convenzioni di scrittura sono elementi aritmetici. La classificazione multipla e la seriazione come l'inclusione di classi (relazione parte-intero), le corrispondenze e le trasformazioni (Piaget \& Voelin, 1980) sono schemi di operazioni cognitivo-psicologiche. Ambedue queste dimensioni, che rientrano nell'aritmetica e nella psicologia cognitiva, devono essere tematizzate ed esercitate durante l'intero arco scolastico.

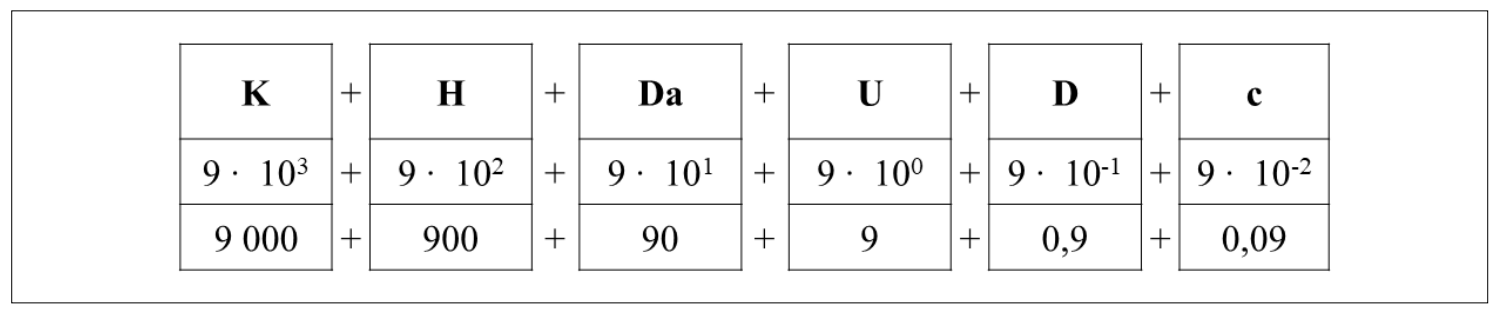

Figura 5. Esempio delle varie espressioni del numero 9 999,99.

La Figura 5 spiega la relazione fra i segni simbolici, mostra forme di rappresentazione e di logica che 
operano in modo astratto. Ma se si vuole superare un'impostazione statica, occorre cambiare il focus. Da questo punto di vista Barrow afferma:

«I matematici hanno estratto il progresso matematico dagli esempi specifici che hanno usato per la loro introduzione e studiano i concetti di "numero", "forma" o "distanza" come enti astratti. Lo fanno dirigendo la loro attenzione alle operazioni che cambiano i numeri, non sui numeri stessi».

(Barrow, 1999, p. 164, traduzione dell'autore)

La didattica dinamica della matematica orientata all'intuizione dovrebbe dunque lavorare con vari aspetti del numero, rappresentazioni e segni, con il «calcolo ragionato» e con l'«astrazione riflessiva».

Nella Tabella 1 sono illustrate le connessioni tra i livelli cognitivi secondo Piaget (1977a, 1977b) e Piaget et al. (1990), i livelli cognitivi della comprensione del sistema di numerazione decimale posizionale secondo Ross (1986) e Herzog et al. (2019), i modi di agire nelle varie fasi di insegnamento, le diverse forme di rappresentazione e i cicli della formazione secondo il Piano di studio Lehrplan $21^{3}$ (concordato fra i cantoni della Svizzera tedesca). Il contenuto della Tabella 1 è orientato verso il concetto dell' «accelerazione cognitiva» (Adey, 2008).

\begin{tabular}{|c|c|c|c|}
\hline \multicolumn{2}{|c|}{ Piano 21 (2016, versione modificata) } & \multicolumn{2}{|c|}{ Psicologia dello sviluppo } \\
\hline Ciclo Età (a.) & Numero e variabili & $\begin{array}{l}\text { Livello } \\
\text { (Ross, 1986; Herzog et al., 2019) }\end{array}$ & $\begin{array}{l}\text { Teoria del morfismo } \\
\text { (Piaget et al., 1990) }\end{array}$ \\
\hline $\begin{array}{l}1 \\
\text { da } \\
4\end{array}$ & $\begin{array}{l}\text { MA.1.A1 Operare e nominare } \\
\text { c) capire e usare i concetti: per, } \\
\text { maggiore di, uguale a, minore } \\
\text { di, pari, dispari, completare, } \\
\text { dimezzare, raddoppiare, decine, } \\
\text { unità e i simboli, }<_{,}>\text {. } \\
\text { Conoscere i numeri naturali } \\
\text { fino a } 100, \text { leggerli e scriverli. }\end{array}$ & $\begin{array}{l}\text { Livello } 1 \text { II numero intero (con } \\
2 \text { cifre) rappresenta l'insieme } \\
\text { degli oggetti (tutte le } 25 \\
\text { barrette). } \\
\text { Livello } 2 \\
\text { Numeri di due cifre rappresen- } \\
\text { tano l'intero insieme ( } 25 \\
\text { barrette). II bambino "ricerca" } \\
\text { significati per le singole cifre. }\end{array}$ & $\begin{array}{l}\text { Livello intramorfico } \\
\text { (preoperativo) } \\
\text { Solo relazioni fra stati } \\
\text { osservabili (5-6 anni). }\end{array}$ \\
\hline $\begin{array}{l}8 \\
\text { anni }\end{array}$ & $\begin{array}{l}\text { MA.1.C2 Matematizzare e } \\
\text { rappresentare } \\
\text { c) sanno descrivere il significato } \\
\text { delle cifre nel sistema posizio- } \\
\text { nale (es: } 5 \text { barrette decine e } 7 \\
\text { cubi-unità rappresentano 57). }\end{array}$ & $\begin{array}{l}\text { Livello } 3 \\
\text { I numeri di due cifre rappresen- } \\
\text { tano l'intero insieme di } 25 \\
\text { oggetti. Le singole cifre } \\
\text { significano decine o unità. II } \\
\text { bambino conosce però in modo } \\
\text { solo impreciso come ciò }\end{array}$ & 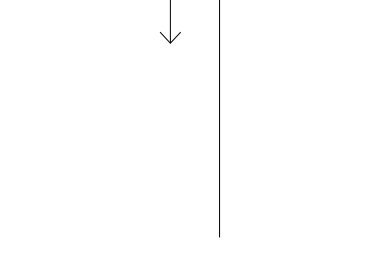 \\
\hline 2 & $\begin{array}{l}\text { MA.1.C2 Matematizzare e } \\
\text { rappresentare } \\
\text { 2) sanno rappresentare il } \\
\text { significato delle cifre nel } \\
\text { sistema posizionale (es: } 2 \\
\text { tessere-centinaia, } 5 \text { barrette- } \\
\text { decine e } 7 \text { cubi-unità rappre- } \\
\text { sentano 257). }\end{array}$ & $\begin{array}{l}\text { Livello } 4 \\
\text { I numeri di due cifre (o } \\
\text { maggiori) rappresentano } \\
\text { l'intero insieme di } 25 \text { o più } \\
\text { oggetti. } \\
\text { I singoli posti sono parti di } \\
\text { numeri interi. Appartengono ai } \\
\text { gruppi delle decine e unità. } \\
\text { L'intero è uguale alla somma } \\
\text { delle parti. }\end{array}$ & $\begin{array}{l}\text { Livello intermorfico } \\
\text { (operazioni concrete) } \\
\text { Coordinamento sistematico } \\
\text { della corrispondenza (tessere, } \\
\text { numeri, posizioni), soprattutto } \\
\text { di stati ed eventi osservabili } \\
\text { (6-10 anni). }\end{array}$ \\
\hline
\end{tabular}

3. È possibile visionare il Piano di studio 21 (Lehrplan21) al sito https://www.lehrplan21.ch. 


\begin{tabular}{|c|c|c|c|}
\hline $\begin{array}{l}\text { da } \\
9\end{array}$ & $\begin{array}{l}\text { MA.1.B3 Esplorare e argomen- } \\
\text { tare } \\
\text { c) per l'esplorazione di strutture } \\
\text { aritmetiche possono essere utili } \\
\text { tavole particolari ("Stellenwert- } \\
\text { tafel", esempio: posizionare e } \\
\text { spostare tessere sulla tavola). }\end{array}$ & & \multirow{4}{*}{$\begin{array}{l}\text { Livello transmorfico } \\
\text { Distacco dagli stati osservabili e } \\
\text { operazioni. } \\
\text { Costruzione di un sistema } \\
\text { astratto operativo, cognitivo. } \\
\qquad \downarrow \\
\text { Sistemi posizionali vengono } \\
\text { costruiti algebricamente (da } 11 \\
\text { anni). }\end{array}$} \\
\hline $\begin{array}{l}12 \\
\text { anni }\end{array}$ & $\begin{array}{l}\text { MA.1.B.1 Esplorare e argomen- } \\
\text { tare } \\
\text { i) sanno stabilire connessioni } \\
\text { aritmetiche mediante variazione } \\
\text { sistematica di numeri, valori } \\
\text { posizionali e operazioni } \\
\text { e compiere osservazioni }\end{array}$ & $\begin{array}{l}\text { Nuovo: Le potenze di } 10 \text { sono } \\
\text { messe in tutte le posizioni, } \\
\text { anche nei numeri decimali } \\
\text { vengono ordinate e spiegate (S. } \\
\text { Meyer, 2020b). }\end{array}$ & \\
\hline da & $\begin{array}{l}\text { MA.1.A.1 Operare e nominare } \\
\text { i) capire e usare i concetti: } \\
\text { termine, variabile, incognita, } \\
\text { elevato a, potenza, potenza di } \\
\text { 10, segno (+o-), numero } \\
\text { positivo, numero negativo, } \\
\text { radice (quadrata). } \\
\text { Estensione: capire e usare i } \\
\text { concetti di base, esponente. }\end{array}$ & $\begin{array}{l}\text { Nuovo: le potenze di base g } \\
\text { vengono ordinatamente } \\
\text { posizionate, rappresentate e } \\
\text { spiegate per mezzo di } \\
\text { espressioni algebriche (S. } \\
\text { Meyer, 2020b). }\end{array}$ & \\
\hline $\begin{array}{l}16 \\
\text { anni }\end{array}$ & $\begin{array}{l}\text { MA.1.B.2 Esplorare e argomen- } \\
\text { tare } \\
\text { j) saper verificare espressioni } \\
\text { algebriche mediante inserimen- } \\
\text { to di numeri. }\end{array}$ & & \\
\hline
\end{tabular}

Tabella 1. Piano di studio 21 (Lehrplan21) (dalla scuola dell'infanzia ai nove anni della scuola obbligatoria) e i livelli di sviluppo cognitivo (S. Meyer, 2020b).

Va considerato che nelle classi c'è diversità nei livelli cognitivi: può succedere che bambini e ragazzi vogliano scoprire ed esaminare i numeri naturali, mentre altri hanno già capito il passaggio ai numeri decimali e alle potenze di 10. È importante interpretare queste differenze come normale diversità e risorsa per stimolare il pensiero operativo nel senso della zona di sviluppo prossimale e della co-costruzione (Vygotskij, 1986; Cuomo, 2007; Adey, 2008).

La Tabella 1 serve come orientamento nelle fasi di sviluppo e azione e anche nella determinazione dei cicli del Piano di studio Lehrplan21.

\section{Stimoli per il rituale dell'aperitivo}

I prossimi paragrafi descrivono quali problemi dovrebbero essere affrontati all'inizio del rituale-aperitivo. Occorre rinunciare a stabilire una sequenza sistematica, ma dare spazio alle domande spontanee dei bambini, ai problemi e agli interessi dell'intera classe. Se la classe suggerisse di affrontare più tematiche in un rituale, i gruppi di preparazione potrebbero creare nuovi compiti per rituali futuri.

Il primo passo, detto "borsa fortunata", raffigura a grandi linee i passi della sequenza. Gli obiettivi vengono tuttavia fissati tenendo conto degli interessi e delle necessità degli alunni e degli insegnanti. Con il tempo si forma un ventaglio di traguardi che andranno elaborati, coordinati e mobilitati al fine 
di far sbocciare la comprensione (Deleuze \& Guattari, 1977). La curiosità e il piacere di capire sono le forze motrici del sistema operativo che porta al raggiungimento del traguardo. Sorgono domande del tipo: «Lo sai perché è così? Cosa succede, se si addiziona il numero 9,99 con 0,01? E perché succede questo? Chi conosce un problema che non abbiamo ancora affrontato? Perché questo è vero?». Ponendo le domande e rilanciando le risposte, gli insegnanti curano il crescere della comprensione come un giardiniere cura le proprie piantine.

\section{Rituale-aperitivo: la "borsa fortunata"}

II rituale della "borsa fortunata" è preparato da gruppetti di allievi secondo uno schema coerente. II gruppo è responsabile del rituale, del materiale e delle domande dei compiti di riflessione. Durante la preparazione i bambini possono chiedere consigli agli insegnanti o ai compagni. I contenuti rimangono segreti fin quando le "borse fortunate" dell'intera classe sono determinate. La preparazione dei compiti è anche una forma di preteaching e di prevenzione (Berg, 2013) soprattutto per allievi con deficit nel vocabolario e nelle prassi. Si esplorano gli interessi e le competenze, si acquisiscono informazioni importanti e conoscenze pratiche e si preparano più chiaramente le prossime lezioni per gli insegnanti e gli studenti.

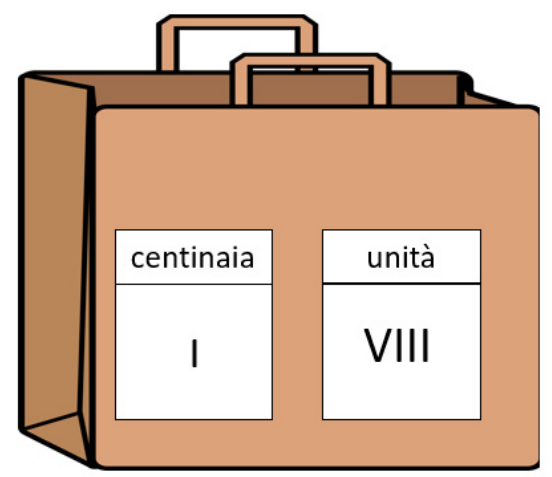

Figura 6. Esempio di materiale di rappresentazione per la borsa fortunata.

La Figura 6 mostra un compito della "borsa fortunata". II gruppo ha scelto di lavorare con un centinaio e 8 unità simbolizzati con numeri romani, come è usanza nei giochi delle carte. L'obiettivo di questo rituale consiste nell'ordinare e interpretare i simboli romani nel sistema decimale posizionale. Un alunno della classe può estrarre le carte dalla borsa fortunata e affiggerle alla lavagna.

Ora i gruppetti discutono in base alle seguenti domande:

1. Di che numero si tratta?

2. Come si scrive questo numero con le cifre indo-arabe?

3. Come potrebbe essere suddiviso il numero dei bastoncini salati a tutta la classe?

Dopo che queste domande sono state discusse nei gruppetti, alcuni relatori dei gruppi mostrano ciò che hanno pensato. Devono scrivere le osservazioni più importanti alla lavagna. L'insegnante incoraggia i gruppi a presentare alla classe tutte le risposte e le riflessioni possibili (Sharingphase, Adey, 2008), loda lo spirito di ricerca della classe ed evita di proposito giudizi del tipo giusto-sbagliato. Eventuali conflitti cognitivi vengono registrati per poi essere affrontati nella "scuola del pensiero", 
che è un colloquio matematico adattivo nel quale i gruppi di allievi e la classe intera esplorano il problema registrato per avanzare nella comprensione logica dell'oggetto matematico.

4. Il quarto passo è dedicato all'astrazione riflessiva ("scuola del pensiero"). I gruppetti discutono in riferimento a domande preparate dagli allievi, di tipo metacognitivo. L'insegnante aiuta nel perfezionamento delle domande.

Riportiamo di seguito alcuni esempi:

- Quali pensieri ti mostrano che il numero trovato sia giusto?

- Quali pensieri ti mostrano che le due carte simboliche sono state convertite fedelmente nella scrittura numerica indo-araba?

- Quali pensieri ti mostrano che il numero di bastoncini salati è stato distribuito correttamente alla classe? Con quali pensieri si può valutare che la distribuzione sia avvenuta correttamente?

Ai gruppetti viene chiesto dall'insegnante di rispondere alle domande nel modo più chiaro possibile. L'insegnante si muove tra i banchi, loda i gruppi per i loro sforzi mentali e ripete le domande di tipo metacognitivo. I pensieri metacognitivi vengono a loro volta presentati alla classe e condivisi con gli alunni. L'insegnante interviene nei casi di giudizi corretto-errato dati dai compagni e rafforza deliberatamente i diversi pensieri e la diversità delle motivazioni (Adey, 2008).

Da questo punto di vista, nell'esperienza di Adey (2008) viene mostrato come le domande metacognitive debbano essere intensamente praticate dagli insegnanti e supervisionate. Spesso tali tipi di domande sorgono in modo inconsapevole, quando si pongono domande dopo l'astrazione empirica (Piaget, 1977a, 1977b), ad esempio: «Cos'hai imparato oggi? È stato facile o difficile? Come avete risolto il compito?» ecc. L'astrazione riflessiva ha bisogno di domande, che richiedono ai bambini non solo di raccontare, ma anche di pensare sul pensare.

Conflitti e problemi possono essere ripresi dai successivi gruppi di preparazione e integrati in un nuovo "borsa fortunata-compito". Su questo l'insegnante attira l'attenzione della classe: esso è il ponte che conduce a successivi rituali e a scuole del pensiero; questa fase finale, nell'accelerazione cognitiva, è detta «bridging» (Adey, 2008).

\section{Tre amici, tre bastoncini salati e valori posizionali}

Con un gioco in stile fumetto gli spettatori vengono introdotti nel significato di valore posizionale con I'aiuto di un abaco scolastico. All'inizio vengono distribuiti tre bastoncini salati a tre persone. Un'altra scena mostra un bastoncino salato su una cartina e due bastoncini salati su un'altra. Una persona interpreta ciò che si è visto nella prima scena, ossia che tre bastoncini salati vengono distribuiti a tre persone. Le due altre persone interpretano l'insieme simbolico in relazione al sistema di valori posizionali come una decina e due unità, dunque come numero 12. I 12 bastoncini salati si distribuiscono secondo un criterio di età in questo modo: la persona più piccola ne riceve 3 , quella media 4 e la più grande 5. Nasce un conflitto cognitivo e sociale, che richiede una soluzione corretta e che nei gruppi di discussione deve essere esaminata e risolta, si veda https://www.youtube.com/watch?v=ZrQaswrCLwC "Three friends, three salt sticks".

La sequenza filmata permette altre interpretazioni e attività. Queste possono scaturire dalla frase: "Quali numeri possono essere creati, se si mantengono i simboli "I" e "II" oppure "II" e "I", ma si assegnano alle cartine altri valori posizionali?» I bambini esplorano in gruppetti nuovi numeri, come per esempio 21, 1200 ecc., usando i bastoncini salati come simboli. 


\section{Quanti bastoncini salati ci sono da mangiare se sono presenti cinque segni simbolici?}

In questo rituale un numero scritto funge sempre da coefficiente del moltiplicando determinato dalla posizione, che rappresenta anche il traguardo di questo rituale. Nei numeri naturali in base 10 possono essere unità, decine, centinaia, i cui prodotti parziali vengono poi addizionati.

Un gruppo potrebbe preparare il rituale-aperitivo insieme all'insegnante: vengono messi nelle scatole, ad esempio, cinque bastoncini salati e tre fogli A4 che determinano le posizioni. Ogni gruppo di lavoro riceve una scatola. II compito è il seguente:

«Quali numeri possono essere trovati con questi cinque bastoncini salati che rappresentano simboli per numeri? Quale sarebbe il numero più piccolo di bastoncini salati per l'aperitivo, quale sarebbe il più grande? Quali numeri tra il più piccolo e il più grande possono essere individuati? Metti i bastoncini salati sui fogli, anche loro si possono suddividere, e scrivi i numeri su un foglio usando le cifre indo-arabe».

Un bambino del gruppo di preparazione potrebbe dare un esempio: con 3 bastoncini salati e 2 fogli di posizione. "Metto 3 bastoncini salati sul foglio delle decine e scrivo il numero 30 sul foglio del protocollo».

\section{Che cosa non va se metto una virgola nel simbolo numerico $5-0,5$ ?}

La percezione e il significato dei segni non stanno fra loro in un rapporto lineare. II rapporto è determinato dal significato della logica del sistema dei valori posizionali decimali. Questo può essere esaminato mediante il rituale "virgola nel simbolo del numero".

La Figura 7 illustra la rappresentazione del numero decimale 0,5 per mezzo dell'abaco scolastico. Gli alunni hanno imparato che la percezione dei segni non è uguale al significato dei segni e alla logica del sistema posizionale. Hanno inoltre già sperimentato con i numeri naturali il cambiamento di posizione. II segno " 5 " può essere collegato quale moltiplicatore in ogni posizione.

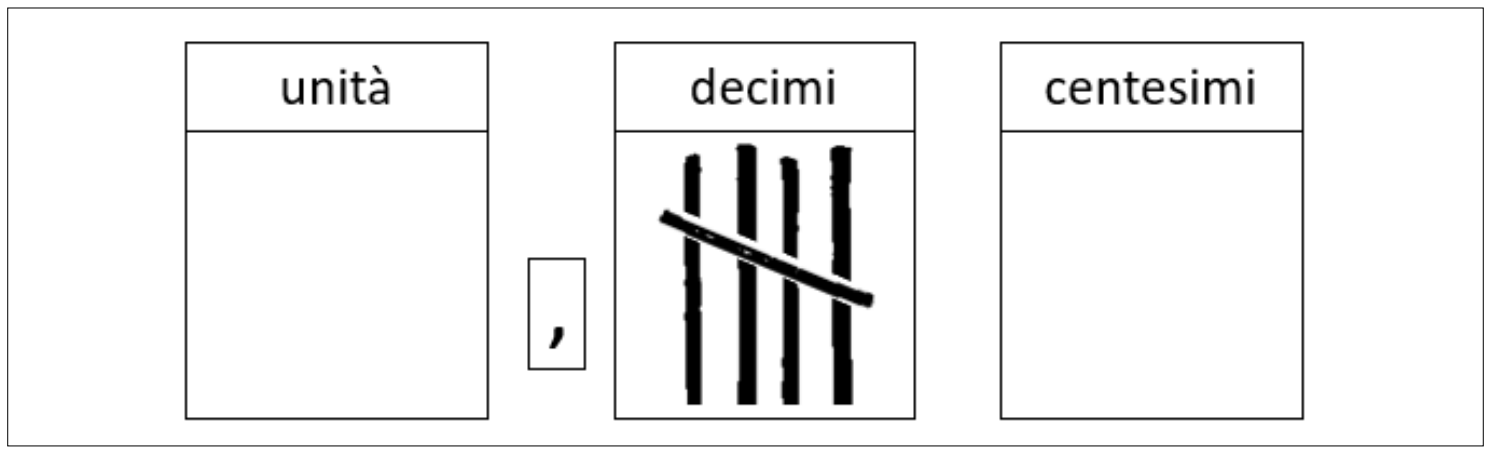

Figura 7. Imparare a percepire e a capire 0,5.

Il traguardo di questo rituale è far sì che gli alunni imparino a sperimentare con rappresentazioni e significati di numeri decimali. 
I gruppi di lavoro ricevono una scatola con il materiale descritto nella Figura 7 e le domande. I| gruppo di preparazione legge le domande e le commenta brevemente. Dopo di che le stesse vengono rielaborate dai gruppi:

1. «Di che numero si tratta? Come lo si legge?»

2. «Come si scrive il numero nel sistema numerico indo-arabo?»

3. «Due bambini come potrebbero ripartire correttamente il numero dei bastoncini indicati?» Alcuni relatori dei gruppi spiegano come nel loro gruppo hanno risolto il problema. Anche in questo caso gli allievi vengono incoraggiati dall'insegnante a comunicare a tutta la classe più risposte e riflessioni possibili (Sharingphase, Adey, 2008). La rinuncia ai giudizi giusto-sbagliato e la lode allo spirito di ricerca della classe sono azioni che l'insegnante deve sempre compiere. I conflitti cognitivi vengono demandati a successive sedute di "scuola del pensiero".

4. Il quarto passo concerne di nuovo l'astrazione riflessiva-metacognitiva. L'insegnante domanda:

- «Quali pensieri ti dimostrano che il numero trovato 0,5 è corretto?»

- «Quali pensieri dimostrano che le carte sono state convertite fedelmente nella scrittura numerica indo-araba?» Se avessi cinque bastoncini salati, ne avrei la metà di dieci. Di conseguenza i cinque bastoncini della figura sono anche la metà di uno. Cioè i cinque bastoncini nella posizione dei decimi sono la metà di un bastoncino salato. Ritornando alla domanda: «Due bambini come potrebbero ripartire correttamente il numero dei bastoncini indicati?», i due bambini ricevono ciascuno la metà di un mezzo bastoncino salato, cioè un quarto di bastoncino.

\section{Prospetiviva}

Siamo dunque giunti alla domanda finale: che cosa significa esattamente un rituale-aperitivo? Seguendo le parole di John Dewey (1993) un rituale-aperitivo si può paragonare a un grammo di esperienza della classe nei confronti di una tonnellata di teoria degli insegnanti: "Un'esperienza, anche umile, può generare e portare teoria in qualsiasi misura, ma una teoria senza riferimento a una qualsiasi esperienza non può nemmeno essere determinata e chiaramente compresa come teoria» (Dewey 1993, p. 193, traduzione dell'autore).

Il rituale che abbiamo presentato, così come l'uso di metodi dinamici, producono esperienze di "aritmetica ludica" e di aritmetica esplorativa e guidata. Vengono svolti meno esercizi ripetitivi e in cambio si crea tempo per l'apprendimento attraverso la pratica (learning-by-doing), per la ricerca in classe (inquiry) e per un rituale comune come quello dell'aperitivo. II rituale, insieme ai metodi dinamici, approfondiscono la scuola del pensiero insieme alla matematica vissuta.

Queste esperienze condurranno a nuove teorie dell'educazione matematica e contribuiranno a sostenere il concetto che la spontaneità e la creatività sono veicoli essenziali per la comprensione dei problemi di matematica.

Traduzione di Gianfranco Arrigo, Società matematica della Svizzera italiana, Lugano, Svizzera.

\section{Bibliografia}

Abrahamson, D., Zolkower, B., \& Stone, E. (2020). Reinventing Realistic Mathematics Education at Berkeley Emergence and Development of a Course for Pre-service Teachers (ICME-13 Monographs). In M. van den 
Heuvel-Panhuizen (Ed.), International Reflections on the Netherlands Didactics of Mathematics: Visions on and Experiences with Realistic Mathematics Education (pp. 255-277). Cham: Springer International Publishing. https://doi.org/10.1007/978-3-030-20223-1 14

Adey, P. (2008). Let's Think! Handbook. A Guide to Cognitive Acceleration in the Primary School. London: GL assessment.

Arrigo, G. (2014). Calcolo mentale-approssimato-strumentale. Bollettino dei docenti di matematica, 68, 53-62.

Barrow, J. D. (1999). Ein Himmel voller Zahlen. Auf den Spuren mathematischer Wahrheit. Reinbek b. Hamburg: Rowohlt.

Berg, J. L. (2013). Preteaching Strategies to Improve Student Learning in Content Area Classes. Intervention in School and Clinic, 49(1), 14-20.

Bodrova, E. (2007). Tools of the Mind. The Vygotskian Approach to Early Childhood Education (2nd ed.). New Jersey: Pearson Education Inc.

Brugger, C., Sidler, A., \& Meyer, S. (2007). Stellenwerte des Zehnersystems verstehen. Rapporto di ricerca inedito (p. 9). Zürich: Hochschule für Heilpädagogik.

Bruner, J. S. (1971). Studien zur kognitiven Entwicklung: eine kooperative Untersuchung am „Center for cognitive studies" der Harvard-Universität. Stuttgart: Klett.

Cuomo, N. (2007). Verso una scuola dell'emozione di conoscere. Il futuro insegnante, insegnante del futuro. Pisa: Edizioni ETS.

Deleuze, G., \& Guattari, F. (1977). Rhizom. Berlin: Merve Verlag.

Devereux, G. (1998). Angst und Methode in den Verhaltenswissenschaften (4. Aufl.). Frankfurt a.M.: suhrkamp taschenbuch verlag.

Dewey, J. (1993). Demokratie und Erziehung: eine Einleitung in die philosophische Pädagogik. Herausgegeben und mit einem Nachwort versehen von Jürgen Oelkers. Weinheim: Beltz.

Dewey, J. (2008). Logik. Die Theorie der Forschung. Frankfurt a.M.: suhrkamp taschenbuch verlag.

Duval, R. (1993). Registres de représentation sémiotique et fonctionnement cognitif de la pensée. Annales de didactique et de sciences cognitives, 5, 37-65.

Duval, R. (2006). Quelle sémiotique pour l'analyse de l'activité et des productions mathématiques? Relime, Número Especial, 45-81.

Flick, U. (2006). Qualitative Sozialforschung (4. vollständig überarbeitete und erweiterte Neuausgabe.). Reinbek b. Hamburg: Rowohlt.

Freire, P. (2011). Pedagogy of the oppressed. New York: Continuum International Publishing Group.

Freudenthal, H. (1977). Mathematik als pädagogische Aufgabe (2., durchgesehene Auflage., Band 1 und 2). Stuttgart: Ernst Klett Verlag.

Freudenthal, H. (1983). Didactical phenomenology of mathematical structures. Dordrecht, the Netherlands: Reidel.

Freudenthal, H. (1991). Revisiting Mathematics Education. China Lectures. Dordrecht: Kluwer Academic Publishers. 
Goldstein, S., Princiotta, D., \& Naglieri, J. A. (2015). Handbook of Intelligence: Evolutionary Theory, Historical Perspective, and Current Concepts (2015. Auflage). Springer.

Gupta, A. (2009). Vygotskian perspectives on using dramatic play to enhance children's development and balance creativity with structure in the early childhood classroom. Early Child Development and Care, 179(8), 1041-1054. Routledge. https://doi.org/10.1080/03004430701731654

Gur-Ze'ev, I. (Ed.). (2005). Critical Theory and Critical Pedagogy Today - Toward a New Critical language in Education (Studies in Education). Haifa: University of Haifa. Disponibile in: https://www.academia.edu/ 195758/Critical Theory and Critical Pedagogy Today - Toward a New Critical language in Education (consultato il 10.10.2020).

Herzog, M., Fritz, A., \& Ehlert, A. (2017). Entwicklung eines tragfähigen Stellenwertverständnisses. In A. Fritz, S. Schmidt \& G. Ricken (Eds.), Handbuch Rechenschwäche (3., vollständig überarbeitete u. erweiterte Aufl., pp. 266-285). Weinheim: Beltz Verlag.

Herzog, M., Ehlert, A., \& Fritz, A. (2019). Development of a Sustainable Place Value Understanding. In A. Fritz, V. G. Haase \& P. Räsänen (Eds.), International Handbook of Mathematical Learning Difficulties: From the Laboratory to the Classroom (pp. 561-579). Cham: Springer International Publishing. https://doi.org/10.100 7/978-3-319-97148-3 33

Inhelder, B., Sinclair, H., \& Bovet, M. (1974). Apprentissage et structures de la connaissance. Paris: Presses Universitaires de France.

Johann, M. (2002). Pumucklzahlen am Abakus. Einsatz des Abakus beim Aufbau des Zahlbewusstseins und des Zahlwortschatzes. Grundschulunterricht, 6, 1-5.

Johann, M., \& Matros, N. (2003). Wechselspiele - Kreatives Rechnen am Schulabakus (2. Auflage). Weinheim: Beltz.

Kamii, C. (1985). Young Children Reinvent Arithmetic. New York: Teachers College Press.

Kamii, C. (1994). Young children continue to reinvent arithmetic. 3rd Grade. New York: Teachers College Press.

Kamii, C. (2000). Number in preschool \& kindergarten (8th Printing.). Washington: National Association for the Education of Young Children.

Kamii, C. (2004). Young Children Continue to Reinvent Arithmetic. 2nd Grade (2nd ed.). New York: Teachers College Press.

Kamii, C. (2005). Teaching arithmetic to low-performing, low-SES first graders. Journal of Mathematical Behavior, 24, 39-50.

Klafki, W. (1996). Neue Studien zur Bildungstheorie und Didaktik. Zeitgemässe Allgemeinbildung und kritisch-konstruktive Didaktik (5. Auflage.). Basel: Beltz Verlag.

Lenné, H. (1969). Analyse der Mathematikdidaktik in Deutschland. Stuttgart: Ernst Klett Verlag.

Lurija, A. R., \& Judowitsch, F. Ja. (1982). Die Funktion der Sprache in der geistigen Entwicklung des Kindes. Frankfurt a.M.: Ullstein.

McCabe, U., \& Farrell, T. (2020). Play, pedagogy and power: a reinterpretation of research using a Foucauldian lens. International Journal of Early Years Education, published online, 1-13. Routledge. https://doi.org/10.1 $\underline{080 / 09669760.2020 .1742669}$

Meyer, H. (2000). Unterrichtsmethoden II: Praxisband (9. Aufl.). Berlin: Cornelsen Scriptor. 
Meyer, S. (2017). Mathematik-Kurz-Test (MKT) 1-9. Flexible Interviews und Blitzrechnen (FI-B). Disponibile in: https://www.hfh.ch/de/unser-service/shop/produkt/mathematik kurztest mkt 19 (consultato il 12.10.2020).

Meyer, S. (2019). "Ich bin dein Taschenrechner" - Kritische Exploration und Rollenspiel. Disponibile in: https:// flexiinterview.blogspot.com/2019/05/ich-bin-dein-taschenrechner-kritische.html (consultato il 10.10.2020).

Meyer, S. (2020a). Bedeutsame Inhalte in der mathematischen Bildung. Was systemische didaktische Analysen bewirken können. Unveröffentlichter Essay, Zürich.

Meyer, S. (2020b). Muster einer Denkschulung - Das Stellenwertsystem. Wahlmodul 207: Das Denken miteinan der schulen - Cognitive Acceleration, Zürich: Interkantonale Hochschule für Heilpädagogik.

Meyer, S., \& Wyder, A. (2017). Mathematik-Kurz-Test (MKT) 1-9. Disponibile in: https://www.hfh.ch/de/un ser-service/shop/produkt/mathematik kurztest mkt 19 (consultato il 10.10.2020).

Moreno, J. L. (1996). Die Grundlagen der Soziometrie. Wege zur Neuordnung der Gesellschaft (Unveränderter Nachdruck der 3. Auflage.). Opladen: Leske + Budrich.

Moreno, J. L. (2007). Theorie der Spontaneität-Kreativität. In H. G. Petzold \& I. Orth (Eds.), Die neuen Kreativitätstheorien. Handbuch der Kunsttherapie. Theorie und Praxis (4. Aufl., Bände 1-II, Band I, pp. 189-202). Bielefeld und Locarno: Edizioni Sirius.

Moser Opitz, E. (2007). Rechenschwäche / Dyskalkulie. Bern: Haupt-Verlag.

Otero, T. M. (2015). Intelligence: Defined as Neurocognitive Processing. In S. Goldstein, D. Princiotta \& J. A. Naglieri (Eds.), Handbook of Intelligence: Evolutionary Theory, Historical Perspective, and Current Concepts (pp. 193-208). New York: Springer.

Piaget, J. (1977a). Recherches sur l'abstraction réfléchissante. 1. L'abstraction des relations logico-arithmétiques. (Études d'épistémologie génétique) (Band 1). Paris: Presses universitaires de France.

Piaget, J. (1977b). Recherches sur l'abstraction réfléchissante. 2. L'abstraction de l'ordre des relations spatiales (Bände 1-2, Band 2). Paris: Presses universitaires de France.

Piaget, J., Henriques, G., \& Ascher, E. (1990). Morphismes et Catégories. Comparer et Transformer. Lausanne: Delachaux et Niestlés.

Piaget, J., \& Szeminska, A. (1975). Die Entwicklung des Zahlbegriffs beim Kinde (Bd. 3). Stuttgart: Ernst Klett Verlag.

Piaget, J., \& Voelin, Cl. (1980). Correspondances et transformations dans le cas de l'intersection. In J. Piaget (Ed.), Recherches sur les correspondances (pp. 121- 131). Paris: Presses Universitaires de France.

Presmeg, N., Radford, L., Roth, W.-M., \& Kadunz, G. (2016). Semiotics in Theory and Practice in Mathematics Education (ICME-13 Topical Surveys). In N. Presmeg, L. Radford, W.-M. Roth \& G. Kadunz (Eds.), Semiotics in Mathematics Education (pp. 5-29). Cham: Springer International Publishing. https://doi.org/10.1007/9783-319-31370-2 2

Reiss, K., \& Schmieder, G. (2005). Basiswissen Zahlentheorie. Berlin: Springer.

Resnick, L. B., Asterhan, C. S. C., \& Clarke, S. N. (2018). Accountable Talk: Instructional dialogue that builds the mind. Educational Practices Series, 29. International Academy of Education. Disponibile in: http://www.iao ed.org (consultato il 12.10.2020).

Ross, S. H. (1986). The Development of Children's Place-Value Numeration Concepts in Grades Two through Five. Gehalten auf der Annual Meeting of the American Educational Research Association, (ERIC Dokument 
Reproduction Service No. ED, 273 482).

Ruflin, A.-L. (2008). Stellenwert unter erschwerten Bedingungen entdecken: verbessert aktiv-entdeckender und problemlösender Mathematikunterricht das Verständnis der Stellenwerte bei lernbehinderten Schülern der Oberstufe? Lavoro per il master, inedito. Zürich: Hochschule für Heilpädagogik.

Schenker, I. (2018). Die didaktische Unterstützung des kindlichen Spielens durch pädagogische Fachkräfte. In I. Schenker (Ed.), Didaktik der Kindertageseinrichtungen. Eine systemisch-konstruktivistische Perspektive (pp. 250-268). Weinheim: Beltz Juventa.

Schuler, M. (2004). Neue Erfahrungen mit dem Zehnerübergang. Unveröff. Modul-Leistungsnachweis. Zürich: Interkantonale Hochschule für Heilpädagogik.

Storch, M. (1996). Kreativität und Psychodrama. Vortrag gehalten auf der 53. Psychotherapie-Seminar vom 22. bis 27. September 1996, Freudenstadt. Disponibile in: https://www.google.com/url?sa=t\&rct= $j \& q=\& e s r c=s \&$ source $=$ web $\& c d=\& c a d=r j a \& u a c t=8 \& v e d=2 a h U K E w i 47 \mathrm{Jmfq} 8 z q A h X M i 1$ wKHeSyDG QQFjAAegQIAhAB\&url=https\%3A\%2F\%2Fzrm.ch\%2Fimages\%2Fstories \%2Fdownload\%2Fpdf\%2Fpu blikationen\%2Fpublikation storch 19960927.pdf\&usg=AOVVaw3k4XHuFuofUakmJNRDXKZH (consultato il 10.10.2020).

Vygotskij, L. S. (1986). Denken und Sprechen. Frankfurt a.M.: Fischer.

Walser, H. (2020). Kinematische Geometrie. Atelier gehalten auf der Wintertagung der Gesellschaft für Didaktik der Mathematik CH, Zürich. Disponibile in: http://www.walser-h-m.ch/hans/Vortraege/20200117/index.

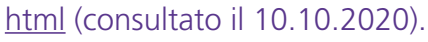

Watzlawick, P. (2017). Menschliche Kommunikation: Formen Störungen, Paradoxien (13., unveränderte Auflage.). Bern: Hogrefe.

Weltgesundheitsorganisation. (2011). ICF-CY. Internationale Klassifikation der Funktionsfähigkeit, Behinderung und Gesundheit bei Kindern und Jugendlichen. Bern: Hans Huber.

Wieser, M., \& Ottomeyer, K. (2000). Spontaneität. In G. Stumm \& A. Pritz (Eds.), Wörterbuch der Psychotherapie (p. 662). Vienna: Springer. https://doi.org/10.1007/978-3-211-99131-2 1804

Wink, J. (2011). Critical pedagogy: notes from the real world (4th ed.). New Jersey: Pearson Education.

Wittgenstein, L. (2013). Bemerkungen über die Grundlagen der Mathematik (Werkausgabe Band 6) (9. Auflage.). Frankfurt a.M.: Suhrkamp Verlag.

Wittmann, E. C. (2002). Grundfragen des Mathematikunterrichts (6. neu bearbeitete Auflage.). Braunschweig: Vieweg.

Wittmann, E. C. (2020). The Impact of Hans Freudenthal and the Freudenthal Institute on the Project Mathe 2000 (ICME-13 Monographs). In M. van den Heuvel-Panhuizen (Ed.), International Reflections on the Netherlands Didactics of Mathematics: Visions on and Experiences with Realistic Mathematics Education (pp. 63-69). Cham: Springer International Publishing. https://doi.org/10.1007/978-3-030-20223-1 4 